\title{
A Valveless Micropump based on Additive Fabrication Technology
}

\author{
Cuong Nguyen Nhu1, Luan Le $\operatorname{Van}^{1,2}$, An \\ Nguyen Ngoc ${ }^{1}$, Tung Thanh Bui ${ }^{1}$, Van Thanh \\ $\mathrm{Dau}^{3}$, and Trinh Chu Duc ${ }^{2}$ \\ ${ }^{1}$ University of Engineering and Technology, Vietnam National University, \\ Hanoi, Vietnam \\ ${ }^{2}$ Vietnam Academy of Science and Technology, Hanoi, Vietnam \\ ${ }^{3}$ Research Group (Environmental Health), Sumitomo Chemical Co. \\ Ltd, Hyogo, Japan \\ E-mail: trinhcd@,vnu.edu.vn
}

\begin{abstract}
A valveless microfluidic pump fabricated by additive fabrication technology is presented. Nozzle/diffuser structures are used to direct the flow of liquid currents inside the PZT-based micropump. A unique design using the collision of two inlet currents helps reduce the back-flow during the suction phase and promotes mixing ability. The micropump is simulated and a positive flow rate is observed at the outlet. The performance of the pump is further investigated at different driving frequencies and voltages. Simulation study on the nozzle/diffuser helps determine an efficient configuration of the nozzle/diffuser of $6^{\circ}$ opening angle. The proposed micropump is suitable for low-cost disposable applications and is capable of delivering a controlled amount of liquid.
\end{abstract}

Keywords: 3D printing, micropump, valveless, finite element method (FEM)

\section{Introduction}

Driven by the need for development in several fields such as biomedical science, microelectronics cooling, micro fluid delivery, etc., research on microvalve and micropump has started in the 1980s and become a vast subject in microelectronics [1], [2]. Micropumps are used to generate a volumetric fluid movement in a microfluidic device. Passive pump and active pump are two main categories that micropumps fall into. Passive pumps require no external energy supply [3]. The surface tension difference between the small droplet at the inlet and the larger droplet at the outlet was employed to convey the fluid sample [4], [5]. The porous media such as paper was also used to transport liquid thanks to the capillary force appearing at the air-liquid interface [6]. These pumps suffer from several shortcomings such as low flow rate, lack of flow rate control and being strong fluid-dependent. On the contrary, active pumps are those which require energy supply. A fluid flow can be generated by applying external electric field 
on an electric double layer (EDL) as in electroosmotic pump (EOP) [7]-[9] or surface tension due to wettability change in electrowetting (EW) pump [10], which use nonmechanical methods. Alternatively, a mechanical movement component can be utilized as an actuator. Membranes or diaphragms are the popular selection for actuation in many of the proposed micropumps. Among them, piezoelectric diaphragms emerge as a notable actuator that can work at both high and low frequencies and different driving voltages, which means a high degree of freedom in controlling the pumping performance.

Rectifying the flow is another important aspect of a micropump. A conventional diaphragm pump consists of two passive check valves connected to an oscillating diaphragm, which creates an oscillating chamber volume [11]. The valves allow the user to control fluid flow in a microchannel by varying a given macroscopic parameter. They can be actuated mechanically, pneumatically, electrokinetically, by phase changes, or by introduction of an external force. Pumps with movable valves, may suffer from problems such as a high pressure drop across the valves and wear and fatigue of the movable parts, resulting in reducing their lifetime and reliability [12], [13].

Pumps without valves (i.e. valveless micropumps) have attracted great interest because their simple structures, have no internal moving parts [14], [15]. That results in a lack of wear due to friction, eliminating sticking effects, and making them highly suitable to integrate into compact lab on a chip applications [12], [16], [17]. To secure reliability, valveless pump structures are highly desirable. Electrohydro-dynamic pump, a simple structure with no movable parts, in which the fluid motion is achieved by using strong electrostatic forces, is a kind of valveless pump [18], [19]. Nozzle/diffuser structures are also used to direct the flow of liquid currents in valveless pump structures [14], [20]. In such kind of structures, studies can be divided in terms of working mechanism, design, actuation mechanism and dimensions.

In this study, we continue to develop a valveless micropump [21] by studying indepth the characteristics of the micropump with numerical method and carrying out improvement on the dimension of the micropump. First, the design and working principle of the micropump is briefly introduced, a prototype of the micropump has been successfully fabricated with $3 \mathrm{D}$ printing technology. The device uses nozzle/diffuser structures to direct the liquid currents. The dynamic simulation of the pump is carried out to study the response of PZT membrane as well as the fluid dynamics inside the pump. The nozzle/diffuser element was parametrically studied to find the configuration that would increase the performance of the micropump.

\section{Design and Working principle}

The device consists of a pump chamber, two inlet channels and one outlet channel as shown in Figure 1 with the detail dimension listed in Table 1. Nozzle/diffuser structures are applied at the ends of the inlet and outlet channels to maximize the performance of the pump effect. Inset in Figure 1 is a zoom-in view at the nozzle/diffuser. The outlet channel is aligned with the central nozzle/diffuser structure but laid perpendicularly to the two inlet currents. The cross section of the outlet is larger than that of the central nozzle.

The pumping performance is determined by the flow behavior at the nozzle, which is formed at the junction of two opposing inlets orthogonally crossing the pumping outlet line. During the suction phase, the nozzle effect amplifies the suction drawing two inlet currents into the pump chamber first due to the close location. Since the inlets are perpendicular to the outlet channel in this area, the coming inlet currents help blocking the backflow from the outlet. At the same time, the nozzle/diffuser at the outlet channel 
Figure 1. Configuration of the micropump. Inset is the zoom-in view at the nozzle/diffuser.

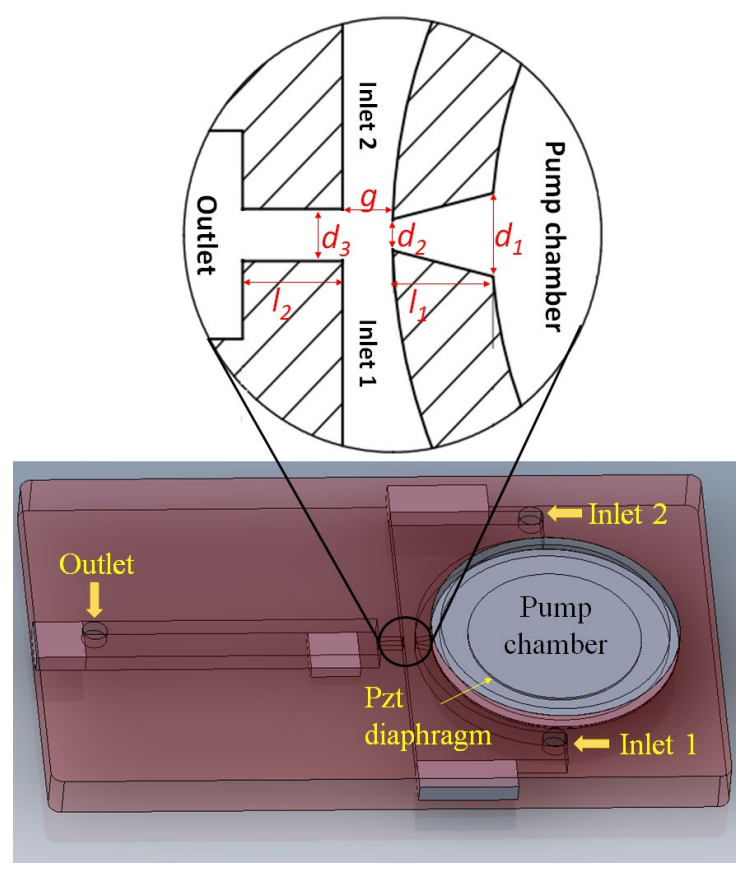

also limits the amount of the liquid in the outlet returning to the pump chamber. During the pump mode, the fluid around the nozzle part of the chamber is pushed through the nozzle. The outgoing mixture will form a high-speed flow that couples with the outlet diffuser whose cross section is larger than that of the chamber's nozzle. Due to the diffuser effect, velocity of the outflow will decrease in the outlet channel.

Table 1. Parameters of the valveless pump

\begin{tabular}{cc}
\hline Parameter & Value $(\mathbf{m m})$ \\
\hline PZT diameter $D$ & 20 \\
$d_{1}$ & 1.6 \\
$d_{2}$ & 0.6 \\
$d_{3}$ & 1 \\
$l_{l}$ & 2 \\
$l_{2}$ & $\mathrm{f} 2$ \\
$g$ & 1 \\
Nozzle thickness & 1.5 \\
Overall thickness & 3.5
\end{tabular}

Thanks to the passive valve element, the proposed pump does not use any moveable part to control the flow. The whole pump body was fabricated with the $3 \mathrm{D}$ printing technology. The simplicity in the structure, fabrication and assembling process makes the proposed pump standout. The PZT membrane actuating the micropump is also a 
Figure 2. Fabricated micropump.

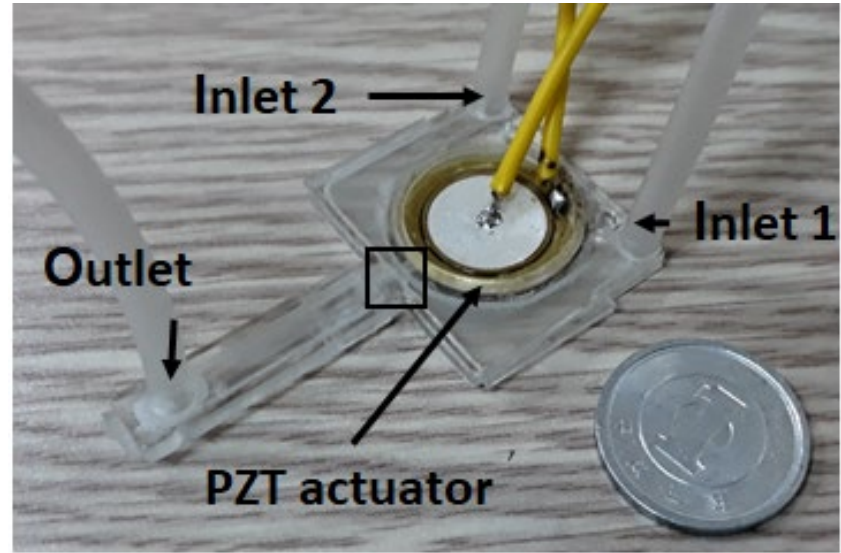

commercial affordable material. These advantages of the proposed pump help minimize the production cost. Figure 2 shows a fabricated prototype of the micropump using a 3D printing system (Connex3 Objet500, Stratasys). The system utilizes polyjet technology in which jets layers of liquid photopolymer as thin as 16 microns to build models and prototypes with extremely complex geometries, fine details, and smooth surfaces [22]. In the future, the experiment on the improved structure will be conducted to validate the simulation results.

\section{Simulation Works}

\subsection{Piezoelectric simulation}

The operation of the PZT membrane was first investigated to determine its response to applied voltages. The deformation captured at a cross-section of the PZT diaphragm in Figure 3 reveals that the diaphragm bends upwards as it is placed under an external voltage. The displacement decreases along the radial direction until it reaches zero at the edge of the diaphragm. This is because the edges of the circular PZT disk are firmly sealed as the top layer of the pump chamber, they were assumed to have no movement. The displacements of the PZT membrane in the X-and Y-directions are trivial. The variation of the displacement and the restriction on displacement at the edge result in the dome shape of the actuator. As the polarity of the voltage is reversed, the piezoelectric material contracts. The membrane bends downwards in dome-shaped form.

Figure 3. Deformation of the membrane
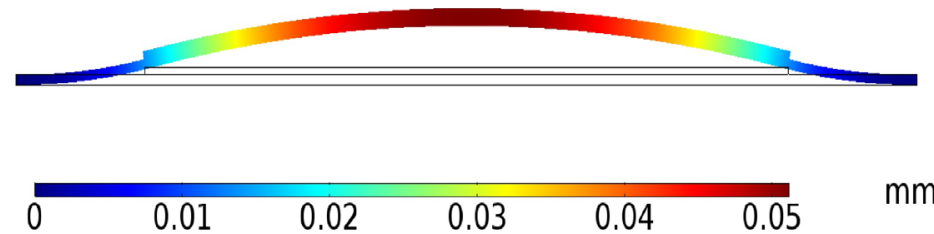
Figure 4. The maximum displacement with different voltages

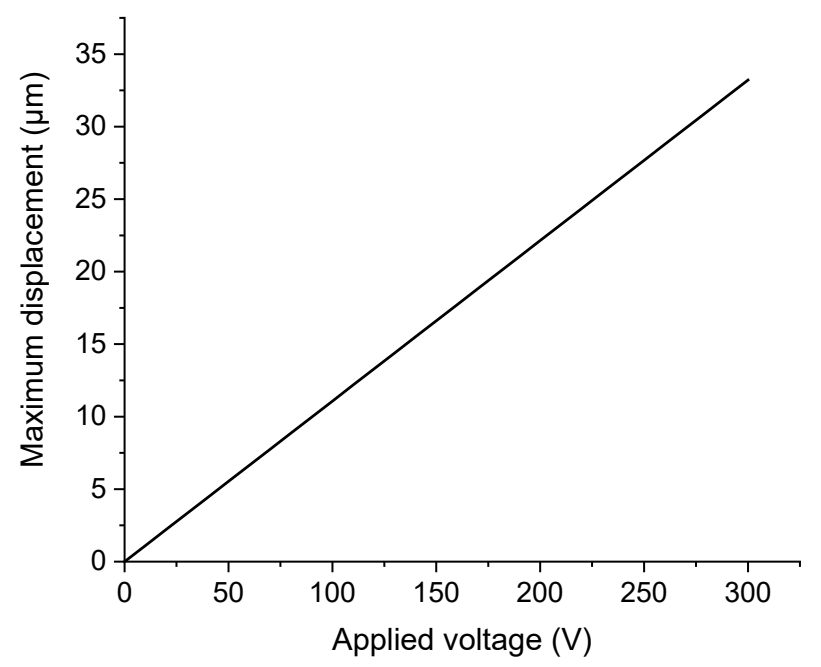

Figure 4 indicates the maximum displacement in z-direction of the membrane i.e. the displacement at the center of the disk. The PZT membrane operates in elastic range for the given condition. It can be observed that the displacement linearly increases with the applied voltage. The displacement is in micrometer range, it is thus guaranteed that the membrane does not strike the chamber bottom.

An insight into the behaviour of the membrane in Figure 5 reveals that its displacement follows the periodic change of the applied voltage. Since the diaphragm serves as both the actuator and as the chamber, the deformation of the actuator leads to the periodic change in the chamber stroke volume. The voltage and frequency can be used as the parameters to control the performance of the pump.

Figure 5. Displacement of the membrane

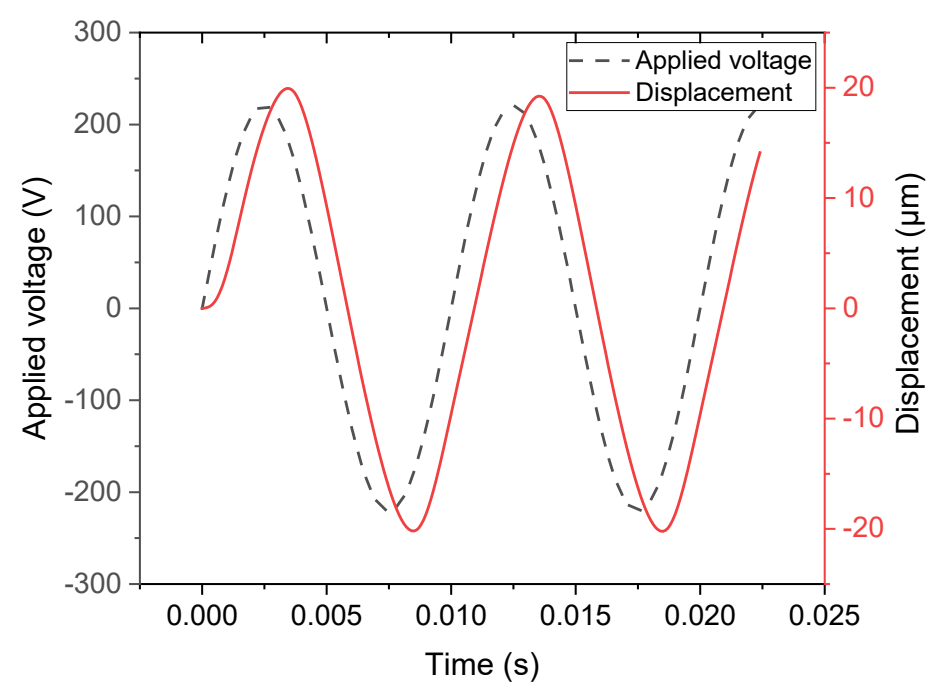




\subsection{Transient FSI simulation}

The fluidic response of the pump was simulated using Fluid-Structure Interaction $(f s i)$, Solid Mechanics (solid) and Electrostatics (es) module in COMSOL Multiphysics. Parameters for material's physical properties are listed in Table 2. Due to the symmetry of the proposed pump, only one haft of the pump was modelled in order to save computational loads and calculating time as in Figure 6. The plastic walls hardly experience any deformation caused by the fluid flow. They were thus removed, and a noslip boundary condition was specified at the walls:

$$
u_{\text {fluid }}=0
$$

The behavior of the single-phase flow of incompressible and Newtonian fluid is governed by the Navier-Stokes equation along with the continuity equation:

$$
\begin{gathered}
\rho\left(\frac{\partial u}{\partial t}+u \cdot \nabla u\right)=-\nabla p+\nabla \cdot\left(\mu\left(\nabla u+(\nabla u)^{T}\right)-\frac{2}{3} \mu(\nabla \cdot u) I\right)+F \\
\nabla \cdot u=0
\end{gathered}
$$

In which, $\mathrm{u}$ is the fluid velocity, $\mathrm{p}$ is the fluid pressure, $\rho$ is the fluid density, and $\mu$ dynamic viscosity and $\mathrm{F}$ is the external forces.

The boundary condition is applied at the surface of the diaphragm where the Fluid-Solid interaction occurs:

Table 2. Physical properties for material used in simulation

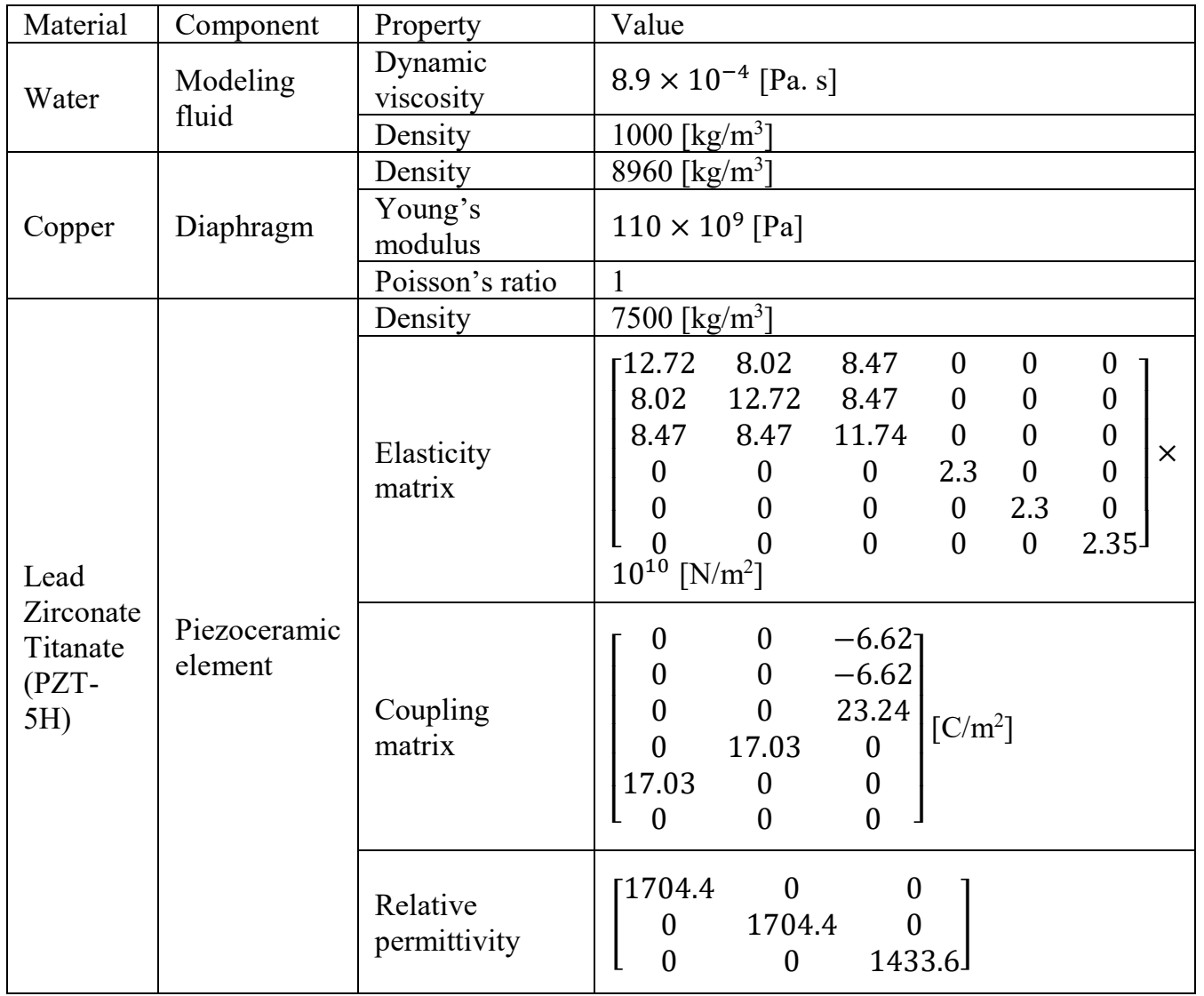




$$
\begin{gathered}
u_{\text {fluid }}=u_{w} \\
u_{w}=\frac{\partial u_{\text {solid }}}{\partial t} \\
\sigma \cdot n=\Gamma \cdot n, \Gamma=\left[-p I+\mu\left(\nabla u_{\text {fluid }}+\left(\nabla u_{\text {fluid }}\right)^{T}\right)\right]
\end{gathered}
$$

where $u_{\text {fluid }}$ and $u_{w}$ is the velocity of the fluid and the solid wall respectively, $u_{\text {solid }}$ is the displacement of the wall, $\sigma$ is the stress tensor, and $n$ is the normal vector to the boundary. The PZT diaphragm is actuated by a time-dependent sinusoidal voltage $V_{a p p}=$ $V_{o} \sin (2 \pi f \times t)$, where $V_{0}$ is the amplitude of the applied voltage, $f$ is the driving frequency. The pump was simulated with the applied voltage of $220 \mathrm{~V}$ and the driving frequency of $100 \mathrm{~Hz}$. It is obtained from Figure 2. that the maximum deformation is approximately $24 \mu \mathrm{m}$.

Figure 6. The symmetric model of the proposed pump

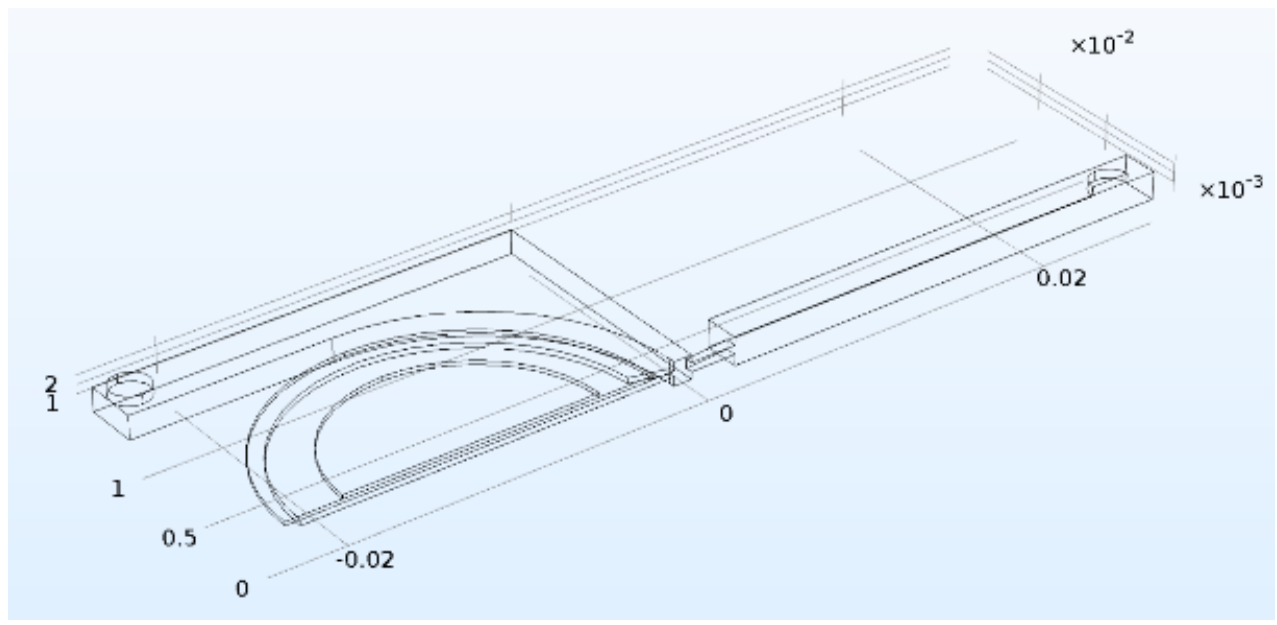

Figure 7. Suction and pumping effects observed at the interrupted nozzle/diffuser structure. (a) Suction phase, (b) Pumping phase.

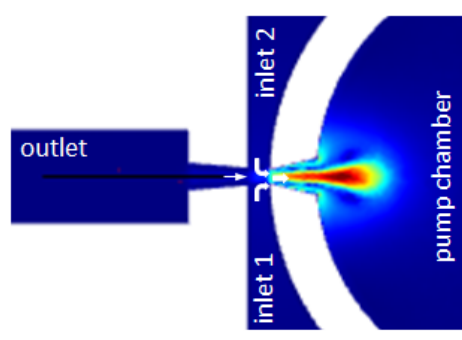

(a) Suction phase

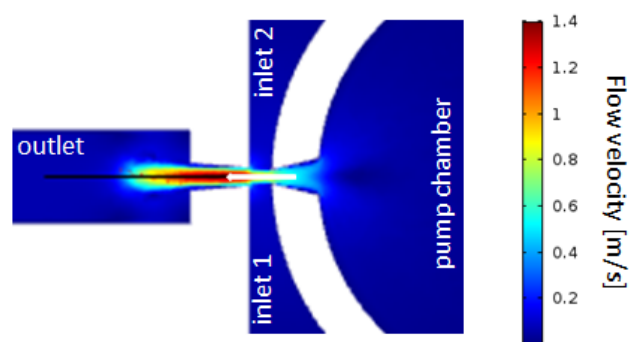

(b) Pumping phase 
The velocity profile of the pump during two working phases are showed in Figure 7 , the length and thickness of the arrows qualitatively represent the magnitude and their directions in the designated channel, confirming the operation of the nozzle/diffuser structure. The increase in the volume of the chamber, created as the membrane moves upwards, results in the pressure drops during the suction phase. An inflow is created at the nozzle drawing liquids deep into the chamber. Inversely, as the chamber pressure increases during the pumping phase, the chamber generates an outflow that couples with the outlet dimension and propagates into the outlet channel.

The instantaneous flow rate at the inlet and the outlet are defined as:

$$
U_{\text {inlet } / \text { outlet }}=\int u_{\text {fluid }} \cdot n d S \text { (7) }
$$

Where $n$ is the normal vector at the surface. The flow rates at the inlet and outlet of

Figure 8. Transient flow rate at the inlet and outlet with $f=100 \mathrm{~Hz}$

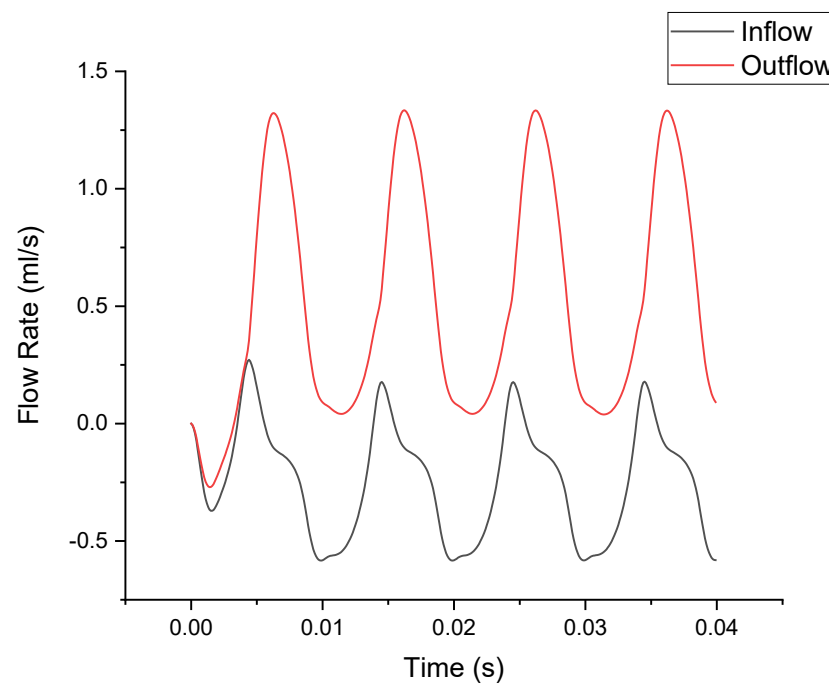

Figure 9. Flow rate at different driving frequencies (simulated)

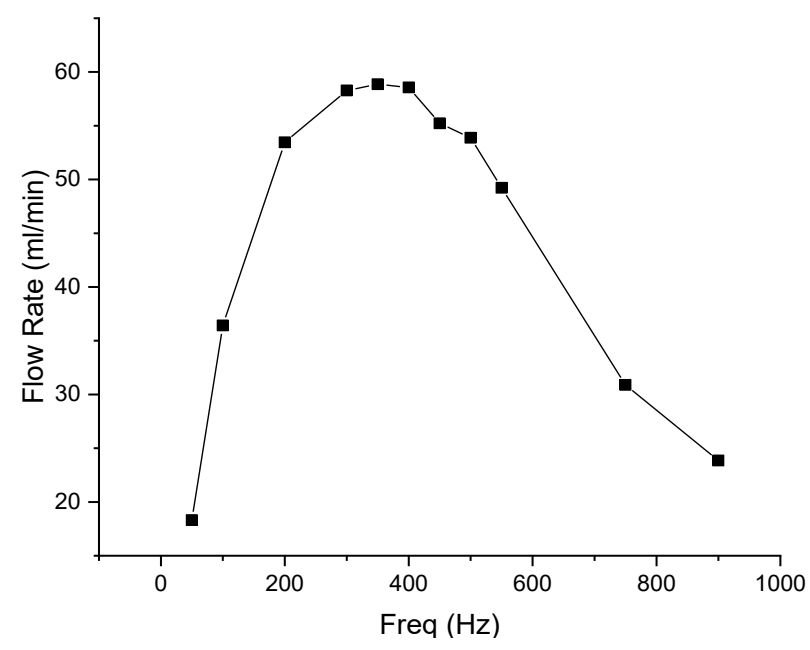


different time step are shown in Figure 8. Flow rate at the inlet is negative over three forth of a period. In other words, the mixture is drawn through the inlet into the pump chamber. The backflow during the pumping phase was minimized by reason of the unique structure. In fact, only in less than a quarter of a period was the fluid drawn back to the source through the inlet. On top of that, the flow rate at the outlet is positive, that is, the fluid is pushed out of the pump. The pumping mechanism is therefore verified.

The amount of fluid conveyed by the proposed pump from the source to the outlet in its operation was calculated by taking the integral of the flow rate at the outlet over time:

$$
V_{\text {pump }}=\int U_{\text {outlet }} d t
$$

The volume of the conveyed fluid within a minute, i.e. the overall flow rate of the pump is presented in Figure 9. It is indicated that at the same applied voltage the performance varies with different frequencies. In other words, frequency appears as a parameter to the flow rate of the pumps. The frequency at which the pump has maximal performance is observed at $400 \mathrm{~Hz}$. After the observed resonant frequency, the pumping capability drop along with the increase of driving frequency.

Figure 10. Simulation model to investigate the diffuser/nozzle dimension

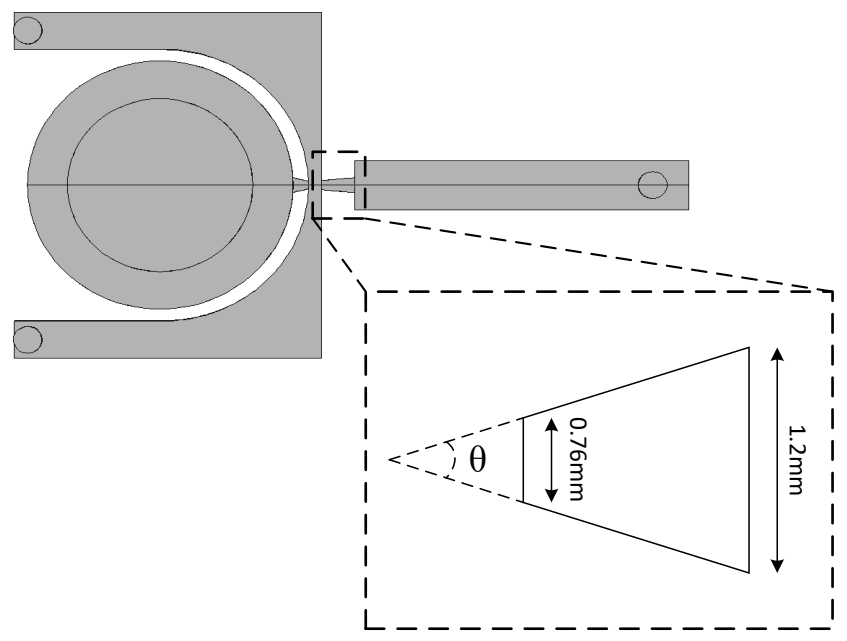

\section{3. Nozzle/Diffuser Element Improvement}

The simulation study on the characteristic of the micropump has proved the working principle of the pump, a positive flow rate has been successfully obtained at the outlet of the pump. We continue to study and improve structure of the pump. Since the function of the nozzle/diffuser is to prevent the backwards flow from the outlet back to the pump. A careful design of the passive element would help block the undesired flow more efficiently, and thus increase the performance of the micropump. In order to study the influence of the nozzle/diffuser element on the performance, a parametric study was conducted on the nozzle/diffuser element. The rectifying element was parametrized. The opening angle of diffuser varies with the angle $\theta$ while the length of the element was kept unchanged as illustrated in Figure 10. The diffusers can be designed as wide angle $\left(60^{\circ}\right.$ to 
Figure 11. Pumped volume in three periods with different opening angles

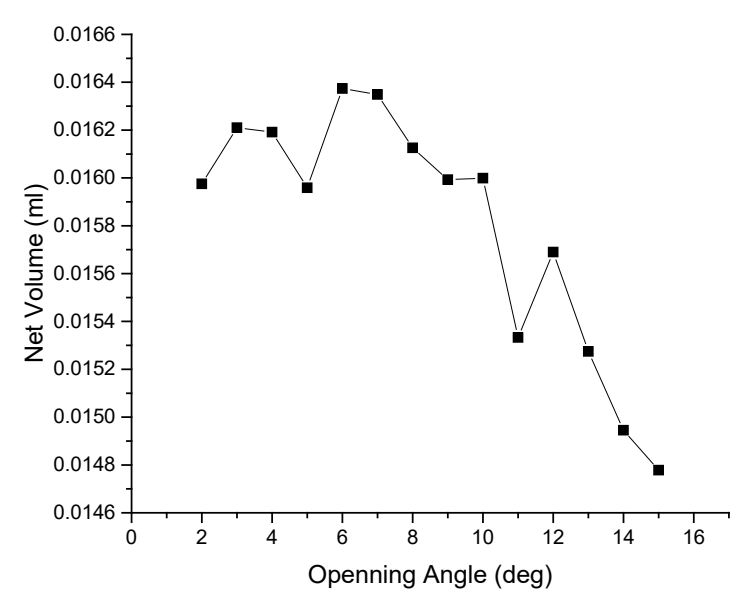

$80^{\circ}$ ) and narrow angle diffuser $\left(4^{\circ}\right.$ to $\left.10^{\circ}\right)$. The wide-angle diffuser was reported to be inefficient due to the boundary layer separation along the diverging direction [23]. The opening angle was incremented from $2^{\circ}$ to $15^{\circ}$ with the $1^{\circ}$ step. The model simulated the systems in three periods. The configuration that produces the highest net volume should be the most efficient one. The results on the pumped fluid in the simulated time is presented in Figure 11. Although result on the pumped volume has some fluctuation, which is likely due to incidental influence of the opening angle on the outlet channel, it can be clearly seen that at the opening angle of $6^{\circ}$ the micropump achieved the highest performance in the studied range. As the opening angle continues to increase, the performance of the pump drops significantly.

The improved micropump is compared with the original pump in Table 3. It can be observed that the performance has been increased from $32.3 \mathrm{ml} / \mathrm{min}$ to $35.2 \mathrm{ml} / \mathrm{min}$, which is approximately $9 \%$. Therefore, the simulation results indicate that the performance of the pump can be effectively improved by changing the angle of the nozzle/diffuser element.

Table 3. Performance comparison of the original and the improved micropump

\begin{tabular}{|l|l|}
\hline Prototype & $\begin{array}{l}\text { Flow } \\
(\mathbf{m l} / \mathbf{m i n})\end{array}$ \\
\hline Original & 32.3 \\
\hline Micropump with new nozzle/diffuser & 35.2 \\
\hline
\end{tabular}

\section{Conclusion}

A valveless microfluidic pump has been developed. Nozzle/diffuser structures are used to direct the flow of liquid currents inside the PZT-based micropump. The performance of the pump has been investigated computationally. The flow rate results at the inlets and outlet demonstrate that the proposed structure for the throat works as expected. The outflow at the outlet is greater than backflow, there exists a positive flow at the outlet. With the same pump configuration, the micropump achieves the highest 
performance at its resonant frequency. From the simulation results, the open angle of the micropump is proposed to be $6 \mathrm{deg}$. With the improved rectifier element, the performance of the micropump has increased approximate by $9 \%$. Further experiments on the micropumps will be carried out in the future.

\section{Acknowledgment}

The authors would like to acknowledge the Vietnamese Ministry of Science and Technology (MOST) for financial support through a project entitled "Development on the aptamer-based and electrical impedance sensing microfluidic system for circulating tumor cells detection", project code NDT.15.TW/16.

\section{References}

[1] F. Abhari, H. Jaafar, and N. A. Md Yunus, A comprehensive study of micropumps technologies, vol. 7, no. 10. 2012.

[2] D. J. Laser and J. G. Santiago, "A review of micropumps," J. Micromechanics Microengineering, vol. 14, no. 6, pp. R35-R64, Jun. 2004.

[3] W. Zhan, C. Li, A. Prof, X. Xiong, P. P. Patra, and P. J. Hu, "COMSOL Simulation of MEMS Piezoelectrically Actuated Micropump,” vol. 14, no. 2, p. $6604,2005$.

[4] G. M. Walker and D. J. Beebe, "A passive pumping method for microfluidic devices," Lab Chip, vol. 2, no. 3, p. 131, Aug. 2002.

[5] P. J. Resto, B. J. Mogen, E. Berthier, and J. C. Williams, “An automated microdroplet passive pumping platform for high-speed and packeted microfluidic flow applications," Lab Chip, vol. 10, no. 1, pp. 23-26, 2010.

[6] X. Wang, J. A. Hagen, and I. Papautsky, "Paper pump for passive and programmable transport," Biomicrofluidics, vol. 7, no. 1, pp. 1-11, 2013.

[7] Y. Takamura, H. Onoda, H. Inokuchi, S. Adachi, A. Oki, and Y. Horiike, "Lowvoltage electroosmosis pump for stand-alone microfluidics devices," Electrophoresis, vol. 24, no. 12, pp. 185-192, Jan. 2003.

[8] J. Ni, F. Huang, B. Wang, B. Li, and Q. Lin, "A planar PDMS micropump using in-contact minimized-leakage check valves.," J. Micromech. Microeng., vol. 20, no. 9, p. 95033, 2010.

[9] X. Wu, P. Ramiah Rajasekaran, and C. R. Martin, “An Alternating Current Electroosmotic Pump Based on Conical Nanopore Membranes," ACS Nano, vol. 10, no. 4, pp. 4637-4643, Apr. 2016.

[10] A. Nisar, N. Afzulpurkar, B. Mahaisavariya, and A. Tuantranont, "MEMS-based micropumps in drug delivery and biomedical applications," Sensors Actuators, B Chem., vol. 130, no. 2, pp. 917-942, 2008.

[11] N.-T. Nguyen, X. Huang, and T. K. Chuan, "MEMS-Micropumps: A Review," J. Fluids Eng., vol. 124, no. 2, p. 384, 2002.

[12] P. Kawun, S. Leahy, and Y. Lai, “A thin PDMS nozzle/diffuser micropump for biomedical applications," Sensors Actuators, A Phys., vol. 249, pp. 149-154, 2016.

[13] E. Stemme and G. Stemme, "A valveless diffuser/nozzle-based fluid pump," Sensors Actuators A. Phys., vol. 39, no. 2, pp. 159-167, 1993.

[14] T. X. Dinh, N. T. M. Le, V. T. Dau, and Y. Ogami, “A dynamic model for 
studying valveless electromagnetic micropumps," J. Micromechanics Microengineering, vol. 21, no. 2, p. 25015, Feb. 2011.

[15] V. T. Dau, T. X. Dinh, Q. D. Nguyen, R. Amarasinghe, K. Tanaka, and S. Sugiyama, "Microfluidic valveless pump actuated by electromagnetic force," Proc. IEEE Sensors, no. Type II, pp. 679-682, 2009.

[16] H. K. Ma, R. H. Chen, N. S. Yu, and Y. H. Hsu, "A miniature circular pump with a piezoelectric bimorph and a disposable chamber for biomedical applications," Sensors Actuators, A Phys., vol. 251, pp. 108-118, 2016.

[17] D. J. Thomas, Z. Tehrani, and B. Redfearn, "3-D printed composite microfluidic pump for wearable biomedical applications," Addit. Manuf., vol. 9, pp. 30-38, 2016.

[18] T. X. Dinh, D. B. Lam, and V. T. Dau, "Mechatronics Jet fl ow in a circulatory miniaturized system using ion wind," Mechatronics, vol. 47, no. September, pp. 126-133, 2017.

[19] V. T. Dau, T. X. Dinh, and T. T. Bui, "Jet flow generation in a circulatory miniaturized system," Sensors Actuators B Chem., vol. 223, pp. 820-826, 2015.

[20] N. Q. Dich, T. X. Dinh, P. H. Pham, and V. T. Dau, "Study of valveless electromagnetic micropump by volume-of-fluid and OpenFOAM," Jpn. J. Appl. Phys., vol. 57201, no. 5, p. 57201, May 2015.

[21] L. Le Van, C. Nguyen Nhu, A. Nguyen Ngoc, T. Bui Thanh, V. Dau Thanh, and T. Chu Duc, "A valveless micropump based on additive fabrication technology," IWNA 2017, 08-11 November, Phan Thiet, Vietnam, pp. 627-630, 2017.

[22] "PolyJet |3D Printing Solutions | Stratasys Direct Manufacturing.".

[23] F. S. Chandika and S. R. Asokan, "Performance evaluation of flat walled diffuser/nozzle micropump for fuel delivery in small gasoline engine," J. Electr. Eng., vol. 12, no. 3, pp. 139-150, 2012. 